\title{
Lowness for uniform Kurtz randomness
}

\author{
Takayuki Kihara ${ }^{1}$ and Kenshi Miyabe ${ }^{2}$ \\ 1 Japan Advanced Institute of Science and Technology, Japan \\ kihara.takayuki.logic@gmail.com \\ 2 Research Institute for Mathematical Sciences, Kyoto University, Japan \\ kmiyabe@kurims.kyoto-u.ac.jp
}

\begin{abstract}
We propose studying uniform Kurtz randomness, which is the uniform relativization of Kurtz randomness. One advantage of this notion is that lowness for uniform Kurtz randomness has many characterizations, such as those via complexity, martingales, Kurtz tt-traceability, and Kurtz dimensional measure.
\end{abstract}

Keywords: algorithmic randomness, lowness for randomness, effective dimension

\section{Introduction}

One of the major topics in algorithmic randomness is "lowness". For a given randomness notion $R, A$ is said to be low for $R$ if every $R$-random set is $R$ random relative to $A$, that is, $A$ does not have enough computational power to derandomize a random set. For instance, lowness for ML-randomness has many characterization such as $K$-trivial, lowness for $K$ and being a base for ML-randomness [14, 9].

Lowness for Schnorr randomness has previously been studied in the literature. Some studies, however, have suggested that uniform Schnorr randomness (the uniform relativization of Schnorr randomness) is the proper relativization because it satisfies van Lambalgen's theorem $[12,13]$ and has natural lowness properties $[7,12,11]$. Similar phenomena have been found for other notions of randomness [1-3]. In particular, the second author and Rute [13] have claimed that uniform relativization is the correct relativization for all randomness notions.

In this paper we study a version of Kurtz randomness. There are already some known results on lowness for Kurtz randomness $[6,16,8]$. Again, however, van Lambalgen's theorem does not hold for Kurtz randomness [7], and it seems that uniform Kurtz randomness (the uniform relativization of Kurtz randomness) is a more natural notion. (Van Lambalgen's theorem for this concept will be studied in another paper.) In this paper, we show that lowness for uniform Kurtz randomness has many characterizations, which advocates for its naturalness.

The overview of this paper is as follows. In Section 3 we introduce uniform Kurtz randomness defined by tests and characterize it via complexity and martingales. In Section 4 we introduce the notion of Kurtz $h$-dimensional measure 
zero where $h$ is an order, and give characterizations via complexity and martingales. In Section 5 we characterize lowness for uniform Kurtz randomness via Kurtz $h$-dimensional measure zero and Kurtz tt-traceability. To prove this, we make use of the svelte tree introduced in Greenberg-Miller [8].

\section{Preliminary}

We say that $n>0$ is the index of a finite set $\left\{x_{1}, \cdots, x_{r}\right\}$ of natural numbers if $n=2^{x_{1}}+2^{x_{2}}+\cdots+2^{x_{r}}$, while 0 is the index of $\emptyset$. In the following we often identify a finite set with its index. We also often identify $\sigma \in 2^{<\omega}$ with the natural number $n$ represented by $1 \sigma$ in binary representation. An order is a nondecreasing unbounded function from $\omega$ to $\omega$. We denote the empty string by $\epsilon$.

We recall some results on Kurtz randomness. The reader may refer to [5, $15]$ for details. Let $\mu$ be the uniform measure on the Cantor space $2^{\omega}$. A set $A \in 2^{\omega}$ is weakly 1-random, or Kurtz random, if it is contained in every c.e. open set with measure $1[10]$. A Kurtz null test is a sequence $\{\llbracket f(n) \rrbracket\}$ such that $f: \omega \rightarrow\left(2^{<\omega}\right)^{<\omega}$ is a computable function and $\mu(\llbracket f(n) \rrbracket) \leq 2^{-n}$. A set is Kurtz random if and only if it passes all Kurtz null tests [17]. A computable measure machine is a prefix-free machine $M$ such that $\mu(\llbracket \operatorname{dom} M \rrbracket)$ is computable [4].

\section{Uniform Kurtz randomness}

Uniform relativization [13] of Kurtz randomness gives the following. Let $\tau$ be the class of open sets on $2^{\omega}$. A partial function $f: \subseteq 2^{\omega} \rightarrow \tau$ is computable if there is a partial computable function $\psi: \subseteq 2^{\omega} \times \omega \rightarrow\left(2^{<\omega}\right)^{<\omega}$ such that $f(Z)=\bigcup_{n \in \omega} \llbracket \psi(Z, n) \rrbracket$ for each $Z$. If such a function $\psi$ is total, then $f$ is also called total.

Definition 1. A uniform Kurtz test is a total computable function $f: 2^{\omega} \rightarrow \tau$ such that $\mu(f(Z))=1$ for all $Z \in 2^{\omega}$. A set $B \in 2^{\omega}$ is Kurtz random uniformly relative to $A \in 2^{\omega}$ if $B \in f(A)$ for each uniform Kurtz test $f$.

Definition 2. A uniform Kurtz null test is a computable function $f: 2^{\omega} \times \omega \rightarrow$ $\left(2^{<\omega}\right)^{<\omega}$ such that, for each $Z \in 2^{\omega}$ and $n \in \omega, \mu(\llbracket f(Z, n) \rrbracket) \leq 2^{-n}$. For a fixed set $X$, we also say that $\{\llbracket f(X, n) \rrbracket\}_{n \in \omega}$ is a Kurtz null test uniformly relative to $X$.

Proposition 1. The following are equivalent for $A \in 2^{\omega}$ and $N \subseteq 2^{\omega}$.

(i) $2^{\omega} \backslash N=f(A)$ for a uniform Kurtz test $f$.

(ii) $N=\bigcap_{n} \llbracket g(A, n) \rrbracket$ for a uniform Kurtz null test $g$.

(iii) A tt-computes a sequence $\left\{C_{n}\right\}$ of finite sets of strings such that $\mu\left(\llbracket C_{n} \rrbracket\right) \leq$ $2^{-n}$ and $N=\bigcap_{n} \llbracket C_{n} \rrbracket$. 
Proof. (i) $\Longleftrightarrow$ (ii) is straightforward.

(ii) $\Rightarrow$ (iii): This is because $\{g(A, n)\}_{n \in \omega}$ is truth-table reducible to $A$.

(iii) $\Rightarrow$ (ii): Let $\Phi$ be a truth-table functional such that $\Phi(A, n)=C_{n}$. Then we can effectively check whether or not $\mu(\llbracket \Phi(Z, n) \rrbracket) \leq 2^{-n}$ because $\llbracket \Phi(Z, n) \rrbracket$ is clopen. If not, we define $\Psi(Z, n)=\emptyset$ and, otherwise, set $\Psi(Z, n)=\Phi(Z, n)$. Then $\Psi$ is a uniform Kurtz null test. Moreover, $\Psi(A, n)=\Phi(A, n)=C_{n}$ for each $n$ and $\bigcap_{n} \llbracket \Psi(A, n) \rrbracket=\bigcap_{n} \llbracket C_{n} \rrbracket$.

Remark 1. If we drop uniformity from the definition of Kurtz tests, the truthtable reducibility $\leq_{t t}$ in (iii) is changed into Turing reducibility $\leq_{T}$. Formally, for every $A \in 2^{\omega}$ and $N \subseteq 2^{\omega}$, (i') its complement $2^{\omega} \backslash N$ is $A$-c.e. open with measure 1, if and only if (ii') $N$ is the intersection of a Kurtz null test relative to $A$ if and only if (iii') $A$ computes a sequence $\left\{C_{n}\right\}$ of finite set of strings such that $\mu\left(\llbracket C_{n} \rrbracket\right) \leq 2^{-n}$, and $N=\bigcap_{n} \llbracket C_{n} \rrbracket$.

We give characterizations of uniform Kurtz randomness via machines and martingales. Recall the following characterization of Kurtz randomness: A set $X$ is not Kurtz random if and only if there is a computable measure machine $M$ and a computable function $f$ such that, for all $n, K_{M}(X \uparrow f(n))<f(n)-n$ if and only if there are a computable martingale $d$ and a computable order $h$ such that $d(X\lceil n)>h(n)$ for all $n[17,6]$.

Proposition 2. The following are equivalent for sets $A$ and $B$.

(i) A is not Kurtz random uniformly relative to $B$.

(ii) There are an oracle prefix-free machine $M$ and a computable function $h$ such that $Z \mapsto \mu\left(\operatorname{dom} M^{Z}\right)$ is a computable function and $K_{M^{B}}(A \uparrow h(n))<$ $h(n)-n$ for all $n \in \omega$.

(iii) There are a $\mathbb{Q}$-valuded martingale $d \leq_{t t} B$ and a computable order $h$ such that

$$
d(A\lceil n)>h(n), \text { for all } n \in \omega .
$$

Proof. (i) $\Longleftrightarrow$ (ii) is straightforward.

(i) $\Rightarrow$ (iii): Suppose that there is a uniform Kurtz null test $f$ such that $A \in$

$\bigcap_{n} \llbracket f(B, n) \rrbracket$ and $\sigma \in f(Z, n) \Rightarrow|\sigma|=g(n)$, where $g$ is a strictly increasing computable function with $g(0)=0$. Let $k$ be a computable order such that $k(0)=0$ and $k(n) \geq g(k(n-1))+1$ for all $n \geq 1$.

We construct a $\mathbb{Q}$-valued martingale $d^{Z}$ as follows. Let $d^{Z}(\epsilon)=2$. At stage $n \geq 1$, we define $d(\sigma)$ for $\sigma \in 2^{<\omega}$ such that $g(k(n-1))<|\sigma| \leq g(k(n))$. Note that $g(k(0))=0$. For each $\tau \in f(Z, k(n-1))$, let $a(\tau)$ be the number of strings $\rho \in f(Z, k(n))$ such that $\tau \prec \rho$. For each $\tau \notin f(Z, k(n-1))$, let $a(\tau)=0$. Note that $a$ is computable from $Z$. We assume that, for each $\rho \in f(Z, n)$, there is $\tau \in f(Z, n-1)$ such that $\tau \prec \rho$, whence $\sum_{\tau \in f(Z, k(n-1))} a(\tau)=\# f(Z, k(n)) \leq$ $2^{g(k(n))-k(n)}$. Let $\sigma \in 2^{<\omega}$ be such that $g(k(n-1))<|\sigma| \leq g(k(n))$. We define a $\mathbb{Q}$-valued martingale $d^{Z}(\sigma)$ by

$$
d^{Z}(\sigma)= \begin{cases}d^{Z}(\sigma\lceil g(k(n-1))) & \text { if } a(\sigma\lceil g(k(n)))=0 \\ e^{Z}(\sigma) & \text { if there is } \tau \in f(Z, k(n)) \text { such that } \sigma \preceq \tau, \\ \frac{d^{Z}(\sigma\lceil g(k(n-1)))}{2} & \text { otherwise. }\end{cases}
$$


where

$$
e^{Z}(\sigma)=d^{Z}\left(\sigma\lceil g(k(n-1)))\left(\frac{1}{2}+\frac{2^{|\sigma|-g(k(n-1))}}{2 \cdot a(\sigma\lceil g(k(n)))}\right) .\right.
$$

Clearly, $d=d^{B} \leq_{t t} B$.

First we show that $d\left(A\lceil g(k(n)))>\left(\frac{3}{2}\right)^{n}\right.$ for all $n \in \omega$. If $n=0, d(\epsilon)=2>$ $1=\left(\frac{3}{2}\right)^{0}$. By assuming that $d\left(A\lceil g(k(n-1)))>\left(\frac{3}{2}\right)^{n-1}\right.$, we have

$$
\begin{aligned}
d\left(A\lceil g(k(n)))=e^{B}(A\lceil g(k(n)))\right. & \geq d\left(A\lceil g(k(n-1)))\left(\frac{1}{2}+\frac{2^{g(k(n))-g(k(n-1))}}{2^{g(k(n))-k(n)+1}}\right)\right. \\
& \geq \frac{3}{2} d\left(A\lceil g(k(n-1)))>\left(\frac{3}{2}\right)^{n} .\right.
\end{aligned}
$$

We define a computable order $h$ by

$$
h(m)=\left\lfloor\frac{1}{2} \cdot\left(\frac{3}{2}\right)^{n-1}\right\rfloor \text { where } g(k(n-1)) \leq m<g(k(n)),
$$

where $\lfloor x\rfloor$ denotes the largest integer not greater than $x$. If $m=0$, then $d(A \uparrow$ $m)=d(\epsilon)=2>1=h(0)=h(m)$. If $m$ satisfies $g(k(n-1))<m \leq g(k(n))$, then

$$
d\left(A\lceil m) \geq \frac{d(A\lceil g(k(n-1)))}{2}>\frac{1}{2} \cdot\left(\frac{3}{2}\right)^{n-1} \geq h(m) .\right.
$$

(iii) $\Rightarrow(\mathrm{i})$ : Assume that $d \leq_{t t} B$. Then there is a truth-table functional $\Psi$ such that $\Psi^{Z}$ is a $\mathbb{Q}$-valued martingale for each $Z \in 2^{\omega}$ and $d=\Psi^{B}$. Let $f$ be a computable order such that $h(f(n)) \geq 2^{n}$ for all $n \in \omega$. Consider the following clopen set:

$$
C_{n}^{Z}=\left\{\sigma \in 2^{f(n)}: \Psi^{Z}(\sigma) \geq 2^{n}\right\} .
$$

Then $\mu\left(C_{n}^{Z}\right) \leq 2^{-n}$ for all $n \in \omega$ and $Z \in 2^{\omega}$. Since $\Psi^{B}(A \uparrow f(n))=d(A \uparrow$ $f(n))>h(f(n)) \geq 2^{n}$ for each $n$, we have $A \in \bigcap_{n} C_{n}^{B}$.

\section{Kurtz Dimensional Measure}

In this section, we introduce and give some characterizations of the notion of Kurtz $h$-dimensional measure zero, which will be used in the next section.

Definition 3. For an order $h: \omega \rightarrow \omega$, a set $E \subseteq 2^{\omega}$ is Kurtz $h$-dimensional measure zero if there is a computable sequence $\left\{C_{n}\right\}_{n \in \omega}$ of finite sets of strings such that

$$
E \subseteq \llbracket C_{n} \rrbracket \text { and } \sum_{\sigma \in C_{n}} 2^{-h(|\sigma|)} \leq 2^{-n} \text { for all } n \in \omega .
$$

We also say that $A \in 2^{\omega}$ is Kurtz $h$-dimensional measure zero if $\{A\}$ is Kurtz $h$-dimensional measure zero.

Theorem 1. Let $h$ be any computable order. Then the following are equivalent for a set $A$. 
(i) A is Kurtz h-dimensional measure zero.

(ii) There are a computable martingale $d$ and a computable order $g$ such that

$$
(\forall n \in \omega)(\exists k \in[g(n), g(n+1))) \quad d\left(A\lceil k) \geq 2^{n} \cdot 2^{k-h(k)} .\right.
$$

(iii) There are a computable measure machine $M$ and a computable order $g$ such that

$$
(\forall n \in \omega)(\exists k \in[g(n), g(n+1))) \quad K_{M}(A\lceil k) \leq h(k)-n .
$$

Proof. (i) $\Rightarrow$ (ii): Suppose that $A$ is Kurtz $h$-dimensional measure zero via a sequence $\left\{C_{n}\right\}_{n \in \omega}$. Find $C_{t(n+1)}$ such that all strings contained in $C_{t(n+1)}$ are longer than any strings of $C_{t(n)}$. Moreover, we assume that $\sum_{\sigma \in C_{t(n)}} 2^{-h(|\sigma|)} \leq$ $2^{-2 n-1}$. For each $\sigma$, let $B_{\sigma}$ be a martingale defined by

$$
B_{\sigma}(\tau)= \begin{cases}2^{|\tau|-h(|\sigma|)} & \text { if } \tau \preceq \sigma \\ 2^{|\sigma|-h(|\sigma|)} & \text { if } \sigma \succ \tau \\ 0 & \text { otherwise. }\end{cases}
$$

Then $d=\sum_{n} \sum_{\sigma \in C_{t(n)}} 2^{n} B_{\sigma}$ is a computable martingale with the initial capital

$$
\sum_{n} \sum_{\sigma \in C_{t(n)}} 2^{n-h(|\sigma|)} \leq \sum_{n} 2^{-n-1}=1 .
$$

Define $g$ to be a computable order such that the length of every string in $C_{t(n)}$ is contained in $[g(n), g(n+1))$. Then, for all $n \in \omega$, there is a $k \in[g(n), g(n+1))$ such that $A\left\lceil k \in C_{t(n)}\right.$, that is,

$$
d\left(A\lceil k) \geq 2^{n} B_{A \mid k}\left(A\lceil k)=2^{n} \cdot 2^{k-h(k)} .\right.\right.
$$

(ii) $\Rightarrow$ (iii): By our assumption, for every $n \in \omega$, there is $k \in[g(n), g(n+1))$ such that $d\left(A\lceil k) \geq 2^{n} 2^{k-h(k)}\right.$. Consider the following clopen set:

$$
C_{n}=\left\{\sigma \in 2^{<\omega}:|\sigma| \in[g(2 n), g(2 n+1)) \text {, and } d(\sigma) \geq 2^{2 n} 2^{|\sigma|-h(|\sigma|)}\right\} .
$$

Let $D_{n}$ be an antichain generating $C_{n}$. Then

$$
\sum_{\sigma \in D_{n}} 2^{n-h(|\sigma|)} \leq \sum_{\sigma \in D_{n}} 2^{n-h(|\sigma|)} \frac{2^{-2 n} d(\sigma)}{2^{|\sigma|-h(|\sigma|)}}=2^{-n} \sum_{\sigma \in D_{n}} 2^{-|\sigma|} d(\sigma) \leq 2^{-n} .
$$

Thus, by the KC theorem [5], we can construct a computable measure machine $M$ such that, for each $n \in \omega, K_{M}(\sigma) \leq h(|\sigma|)-n$ for each $\sigma \in D_{n}$. In particular, for all $n \in \omega$, there is $k \in[g(2 n), g(2 n+1))$ such that $K_{M}(A\lceil k) \leq h(k)-n$.

(iii) $\Rightarrow(\mathrm{i})$ : Assume that $K_{M}(A\lceil k) \leq h(k)-n$ for some $k \in[g(n), g(n+1))$. Consider the sequence $\left\{C_{n}\right\}_{n \in \omega}$ of clopen sets defined by

$$
C_{n}=\left\{\sigma \in 2^{<\omega}:|\sigma| \in[g(n), g(n+1)), \text { and } K_{M}(\sigma) \leq h(|\sigma|)-n\right\} .
$$

Then $A \in \bigcap_{n} C_{n}$, and

$$
\sum_{\sigma \in C_{n}} 2^{-h(|\sigma|)} \leq 2^{-n} \sum_{\sigma \in C_{n}} 2^{-K_{M}(\sigma)} \leq 2^{-n} .
$$

Hence, $A$ is Kurtz $h$-dimensional measure zero. 


\section{Lowness for Uniform Kurtz Randomness}

In this section, we give characterizations of lowness for uniform Kurtz randomness. A set $A \in 2^{\omega}$ is said to be low for uniform Kurtz randomness if $X \in 2^{\omega}$ is uniform Kurtz random relative to $A$ whenever $X$ is Kurtz random. A set $A \in 2^{\omega}$ is said to be low for uniform Kurtz tests if $f(A)$ includes a Kurtz test for every uniform Kurtz test $f$. For a given order $p$, a computable trace with bound $p$ is a computable sequence $\left\{D_{n}\right\}_{n \in \omega}$ of finite sets of strings such that $\# D_{n} \leq p(n)$ for each $n \in \omega$. A computable trace $\left\{D_{n}\right\}_{n \in \omega}$ Kurtz-traces a function $f: \omega \rightarrow \omega$ if there is a computable sequence $\left\{l_{n}\right\}_{n \in \omega}$ of natural numbers such that

$$
(\forall k \in \omega)\left(\exists n \in\left[l_{k}, l_{k+1}\right)\right) \quad f(n) \in D_{n} .
$$

A set $A \in 2^{\omega}$ is said to be Kurtz tt-traceable if there is a computable order $p$ such that, for every $f \leq_{t t} A$, there is a computable trace with bound $p$ that Kurtz-traces $f$.

Theorem 2. The following are equivalent for a set $A$.

(i) A is Kurtz h-dimensional measure zero for every computable order $h$.

(ii) $A$ is low for uniform Kurtz tests.

(iii) $A$ is low for uniform Kurtz randomness.

(iv) A tt-computes no infinite subset of a Kurtz random set.

(v) A is Kurtz tt-traceable.

Proof. (i) $\Rightarrow$ (ii): Let $\left\{C_{n}^{A}\right\}_{n \in \omega}$ be a Kurtz null test uniformly relative to $A$, that is, there is a truth table functional $\Psi$ such that $\llbracket \Psi^{A}(n) \rrbracket=C_{n}^{A}$, and $\mu\left(C_{n}^{A}\right) \leq$ $2^{-n}$. Then there is a computable order $u$ such that, for all $Z \in 2^{\omega}$ and all $n \in \omega$, the value $\Psi^{Z \mid u(n)}(n)$ is determined. In particular, $\llbracket \Psi^{A \mid u(n)}(n) \rrbracket=C_{n}^{A}$. Let $h$ be a computable order fulfilling $2^{-h(u(n))} \geq 1 /(n+1)$ for all $n \in \omega$. Assume that $A$ is Kurtz $h$-dimensional measure zero. By our assumption, we have a computable sequence $\left\{D_{n}\right\}_{n \in \omega}$ of finite sets of strings such that $A \in \llbracket D_{n} \rrbracket$ and $\sum_{\sigma \in D_{n}} 2^{-h(|\sigma|)}<1 /(n+1)$ for all $n \in \omega$. Thus, each $\sigma \in D_{n}$ has length greater than $u(n)$, and moreover $D_{n}$ contains at most $k$ strings of length $\leq u(n+k)$, since, otherwise,

$$
\sum_{\sigma \in D_{n}} 2^{-h(|\sigma|)} \geq(k+1) 2^{-h(u(n+k))} \geq \frac{k+1}{n+k+1} \geq \frac{1}{n+1} .
$$

Hence, $D_{n}$ can be viewed as a finite sequence $\left\{\sigma_{k}^{n}\right\}_{k<\left|D_{n}\right|}$ of strings such that the length of each $\sigma_{k}^{n}$ is greater than or equal to $u(n+k)$. Thus, there is $k<\left|D_{n}\right|$ such that $A\left\lceil u(n+k)=\sigma_{k}^{n}\lceil u(n+k)\right.$. Inductively define a computable order $r$ by $r(0)=0$ and $r(n+1)=r(n)+\left|D_{r(n)}\right|$. Now $\rho(k)$ is defined by $\sigma_{k-r(n)}^{r(n)}\lceil u(k)$ for each $k \in[r(n), r(n+1))$. Then

$$
(\forall n \in \omega)(\exists k \in[r(n), r(n+1))) A\lceil u(k)=\rho(k) .
$$


For all $n \in \omega$ and $k \in[r(n), r(n+1))$, define $E_{k} \subseteq 2^{\omega}$ by

$$
E_{k}= \begin{cases}\llbracket \Psi^{\rho(k)}(k) \rrbracket, & \text { if } \mu\left(\llbracket \Phi^{\rho(k)}(k) \rrbracket\right) \leq 2^{-k} \\ \emptyset, & \text { otherwise. }\end{cases}
$$

Note that $|\rho(k)|=u(k)$ implies that $\Psi^{\rho(k)}(k)$ is defined for all $k \in \omega$ by our assumption for $u$. Therefore, $\left\{E_{k}\right\}_{k \in \omega}$ is a computable sequence of clopen sets, and we have

$$
C_{n}^{A} \subseteq \bigcup_{k=r(n)}^{r(n+1)-1} E_{k} \text {, and } \mu\left(\bigcup_{k=r(n)}^{r(n+1)-1} E_{k}\right) \leq 2^{-r(n)+1} \leq 2^{-n+1} .
$$

Consequently, for $B_{n}=\bigcup_{r(n-1)<t<r(n)} E_{t}$, the sequence $\left\{B_{n}\right\}_{n \in \omega}$ is a Kurtz null test such that $\bigcap_{n} C_{n}^{A} \subseteq \bigcap_{n} B_{n}$. In other words, $A$ is low for uniform Kurtz null tests.

(ii) $\Rightarrow$ (iii): Obvious.

(iii) $\Rightarrow$ (iv): Let $I \subseteq \omega^{\leq \omega}$ be the set of (finite or infinite) strings $\sigma \in \omega \leq \omega$ which are strictly increasing, that is, $\sigma(n)<\sigma(n+1)$ for each $n \in \omega$. Let $\operatorname{rng}(\sigma)$ denote the range of $\sigma \in I$, so that $\operatorname{rng}(\sigma)=\{\sigma(n): n<|\sigma|\}$. From now on, we think of each $B \subseteq \omega$ as a strictly increasing string $B^{\star} \in I$, where $B^{\star}(n)$ is the $n$-th least element contained in $B$. For any $\sigma \in I$, we denote by $P^{\sigma}$ all supersets of the subset of $\omega$ obtained from $\sigma$, that is,

$$
P^{\sigma}=\left\{X \in 2^{\omega}: \operatorname{rng}(\sigma) \subseteq X\right\}
$$

Lemma 1. A set $A$ tt-computes an infinite subset of a Kurtz random set if and only if there exists an infinite set $B \leq_{t t} A$ such that the class $P^{B^{\star}}$ contains a Kurtz random set. Moreover, if a set $A$ tt-computes an infinite set $B \subseteq \omega$, then $P^{B^{\star}}$ is a Kurtz null test uniformly relative to $A$.

Proof. The first equivalence clearly holds. Assume that there is a truth-table functional $\Psi$ such that $\Psi^{A}=B$. Inductively define a truth-table functional $\Phi^{Z}(n)$ by $\Phi^{Z}(0)=\Psi^{Z}(0)$, and $\Phi^{Z}(n+1)=\max \left\{\Psi^{Z}(n+1), \Phi^{Z}(n)+1\right\}$ for each $n \in \omega$. Then, $\Phi^{Z}$ defines an infinite set $B(Z)$, for every $Z \in 2^{\omega}$. Moreover, if $B(Z)$ is infinite, then $P^{B(Z)^{\star}}$ is null. Therefore, $Z \mapsto P^{B(Z)^{\star}}$ is a uniform Kurtz null test. Hence, $P^{B^{\star}}=P^{B(A)^{\star}}$ is a Kurtz null test uniformly relative to $A$.

Now, assume that $A$ is low for uniform Kurtz randomness. By Lemma 1, the class $P^{B^{\star}}$ is a Kurtz null test uniformly relative to $A$ for every $B \leq_{t t} A$. By lowness, $P^{B^{\star}}$ contains no Kurtz random set. Again by Lemma 1, $A t t$-computes no infinite subset of a Kurtz random set.

(iv) $\Rightarrow(\mathrm{v})$ : We again use the notation $P^{f}$ for $f \in I$. As in the proof of Lemma 1 , for each increasing total function $f \in I \cap \omega^{\omega}$ we can see that $f \leq_{t t} A$ if and only if $\operatorname{rng}(f) \leq_{t t} A$. We first recall the following property of $P^{f}$. 
Lemma 2 (See Greenberg-Miller [8, Theorem 5.2]). Let $f: \omega \rightarrow \omega$ be a strictly increasing function. Then, no Kurtz null test includes $P^{f}$ if and only if $P^{f}$ contains a Kurtz random set.

The key notion we will use is that of the svelte tree introduced by GreenbergMiller [8]. A finite antichain $A \subseteq \omega^{<\omega}$ is $k$-svelte via a sequence $\left\{S_{n}\right\}_{n \in \omega}$ of finite sets if

$$
S_{m} \subseteq \omega^{k+m}, \# S_{m} \leq 2^{m} \text {, and } \llbracket A \rrbracket \subseteq \bigcup_{m \in \omega} \llbracket S_{m} \rrbracket .
$$

For $A \subseteq \omega^{<\omega}$ and $\sigma \in \omega^{<\omega}$, the conditional weight $\nu(A \mid \sigma)$ is defined by

$$
\nu(A \mid \sigma)=1-\prod_{\tau \in A \cap[\sigma]}\left(1-2^{-|\tau|-|\sigma|}\right) .
$$

Note that $\nu(A)=\bigcup_{\sigma \in A} \mu\left(P^{\sigma}\right)$. A sequence $\left\{S_{n}\right\}_{n \in \omega}$ of finite sets is ordered by weight with respect to $A$ if $\bigcup_{n} S_{n}$ is an antichain and each $S_{n}$ chooses the first $2^{n}$ strings $\tau \in \omega^{k+n}$ by descending order with regard to $\nu\left(A \backslash \bigcup_{m<n} \llbracket S_{m} \rrbracket \mid \tau\right)$.

Lemma 3 (Essentially due to Greenberg-Miller [8, Theorem 3.3]). For a finite antichain $A \subseteq \omega^{<\omega}$ and a natural number $k \in \omega$, if $\mu\left(\bigcup_{f \in \llbracket A \rrbracket} P^{f}\right) \leq$ $2^{-(k+1)}$ holds, then $A$ is $k$-svelte via an ordered sequence by weight with respect to $A$. In particular, such a sequence is effectively calculated from $A$ and $k$.

Given a closed set $Q \subseteq 2^{\omega}$, let $N_{Q} \subseteq \omega^{\omega}$ be the set $\left\{f \in \omega^{\omega} \cap I: P^{f} \subseteq Q\right\}$.

Lemma 4 (See Greenberg-Miller [8, Lemma 4.3]). If $Q \subseteq 2^{\omega}$ is clopen, then we can effectively find a finite antichain $A_{Q} \subseteq \omega^{<\omega}$ such that $N_{Q}=\llbracket A_{Q} \rrbracket$.

We restrict our attention to a bounded subset of $N_{Q}$ for given a closed set $Q$. For each order $u$, we denote by $N_{Q}^{u}$ the set of all $f \in N_{Q}$ such that $|f(n)|=u(n)$ for each $n \in \omega$, where we think of each $f \in N_{Q}$ as a function from $\omega$ into $2^{<\omega}$.

Lemma 5. Assume that $Q$ is a Kurtz null test. Then, for each order $u$, there are a computable trace $\left\{D_{n}\right\}_{n \in \omega}$ with bound $n \mapsto 2^{n}$ and $D_{n} \subseteq \omega^{n}$ for each $n \in \omega$ and a computable sequence $\left\{l_{k}\right\}_{k \in \omega}$ of natural numbers such that

$$
N_{Q}^{u} \subseteq \bigcup_{n=l_{k}}^{l_{k+1}-1} \llbracket D_{n} \rrbracket, \text { for every } k \in \omega .
$$

Proof. Assume that a Kurtz null test $\left\{C_{n}\right\}_{n \in \omega}$ with $\mu\left(C_{n}\right) \leq 2^{-n}$ and $Q=$ $\bigcap_{n} C_{n}$ is given. By Lemma 4 , we can effectively find a sequence $\left\{A_{n}\right\}$ of finite antichains generating $\left\{N_{C_{n}}\right\}$. By the definition of $N_{C_{n}}$, we have $\bigcup_{g \in \llbracket A_{n} \rrbracket} P^{g} \subseteq$ $C_{n}$. Hence, $\mu\left(\bigcup_{g \in \llbracket A_{n} \rrbracket} P^{g}\right) \leq 2^{-n}$. Therefore, by noting the weight with respect to $A_{n}$, we can effectively find a sequence $\left\{S_{m}^{n}\right\}_{m \in \omega}$ confirming that $A_{n+1}$ is $n$-svelte, uniformly in $n$, by Lemma 3 . In other words,

$$
S_{m}^{n} \subseteq \omega^{n+m}, \# S_{m}^{n} \leq 2^{m} \text {, and } N_{C_{n+1}}=\llbracket A_{n+1} \rrbracket \subseteq \bigcup_{m \in \omega} \llbracket S_{m}^{n} \rrbracket .
$$


For each computable order $u$, because $N_{C_{n+1}}^{u}$ is compact, it is covered by $\bigcup_{m<c(n)} S_{m}^{n}$ for some $c(n) \in \omega$. Note that we can effectively find such a $c(n)$, since $N_{C_{n+1}}^{u}$ and $\bigcup_{m} S_{m}^{n}$ are computable. Inductively define $l_{0}=0$, and $l_{n+1}=$ $l_{n}+c\left(l_{n}\right)$ for each $n \in \omega$. For each $k \in \omega$ and each $n \in\left[l_{k}, l_{k+1}\right)$, we define $D_{n}=S_{n-l_{k}}^{l_{k}} \subseteq \omega^{n}$, where $\# D_{n} \leq 2^{n-l_{k}} \leq 2^{n}$. We now have

$$
N_{Q}^{u} \subseteq N_{C_{k}}^{u} \subseteq \bigcup_{m<c\left(l_{k}\right)} \llbracket S_{m}^{l_{k}} \rrbracket=\bigcup_{n=l_{k}}^{l_{k+1}-1} \llbracket D_{n} \rrbracket
$$

for every $k \in \omega$, as desired.

Now, we assume that $A$ tt-computes no infinite subset of a Kurtz random set. For each $g \leq_{t t} A$, we claim the existence of a computable trace with bound $n \mapsto 2^{n+1}$ that Kurtz-traces $g$. Let $\Psi$ be a truth-table functional such that $\Psi(A)=g$, and find a computable order $u$ such that $\Psi(Z\lceil u(n), n)$ is defined for all $n \in \omega$. Then, in particular, $\Psi(A\lceil u(n), n)=g(n)$. Define $f(n)=A\lceil u(n)$ for each $n \in \omega$. By Lemmas 1 and 2, for every order $u$ and every strictly increasing function $f \leq_{t t} A$ with $|f(n)|=u(n)$ for each $n \in \omega$, there is a Kurtz null test $Q \subseteq 2^{\omega}$ such that $P^{f} \subseteq Q$ holds. Note that $P^{f} \subseteq Q$ if and only if $f \in N_{Q}^{u}$. Since $Q$ is a Kurtz null test, we have two sequences $\left\{D_{n}\right\}_{n \in \omega}$ and $\left\{l_{k}\right\}_{k \in \omega}$ in Lemma 5 . Thus, every $h \in N_{Q}^{u}$ is Kurtz $t$-traceable via $\left\{D_{n}\right\}_{n \in \omega}$ and $\left\{l_{k}\right\}_{k \in \omega}$. For each string $\sigma \in\left(2^{<\omega}\right)^{<\omega}$, let $\sigma^{*}$ denote the last value of $\sigma$, that is, $\sigma^{*}=\sigma(|\sigma|-1)$. Note that $\sigma \in D_{n+1} \subseteq\left(2^{<\omega}\right)^{n+1}$ implies that $\Psi\left(\sigma^{*}, n\right)$ is defined, since $\sigma^{*}$ is of length $u(n)$. For $E_{n}=\left\{\Psi(\sigma, n): \sigma \in D_{n+1}\right\}$, the trace $\left\{E_{n}\right\}_{n \in \omega}$ Kurtz-traces $n \mapsto \Psi(h(n), n)$ for all $h \in N_{Q}^{u}$. In particular, $g: n \mapsto \Psi(f(n), n)$ is Kurtz tt-traceable.

$(\mathrm{v}) \Rightarrow(\mathrm{i})$ : Assume that $A$ is Kurtz $t$-traceable via a computable order $n \mapsto$ $2^{p(n)}$. Given a computable order $h$, we can find a computable order $u: \omega \rightarrow \omega$ such that $h(u(n)) \geq p(n)+n+1$ for each $n \in \omega$. By our assumption, we have a computable trace $\left\{D_{n}\right\}_{n \in \omega}$ with $\# D_{n} \leq 2^{p(n)}$ and a computable sequence $\left\{l_{k}\right\}_{k \in \omega}$ of natural numbers, where, for every $k \in \omega$, there is $n \in\left[l_{k}, l_{k+1}\right)$ such that $A\left\lceil u(n) \in D_{n}\right.$. Without loss of generality, we may assume that $D_{n} \subseteq 2^{u(n)}$. Then, for each $\sigma \in D_{n+1}$, define $C_{k}=\bigcup_{n \in\left[l_{k}, l_{k+1}\right)} D_{n}$, for each $k \in \omega$. Note that $A \in \llbracket C_{k} \rrbracket$ for all $k \in \omega$. To estimate the weight of $C_{k}$, we note the following inequality:

$$
\begin{aligned}
\sum_{\sigma \in C_{k}} 2^{-h(|\sigma|)} & =\sum_{n=l_{k}}^{l_{k+1}-1} \# D_{n} \cdot 2^{-h(u(n))} \\
& \leq \sum_{n=l_{k}}^{l_{k+1}-1} 2^{p(n)-h(u(n))} \leq \sum_{n=l_{k}}^{l_{k+1}-1} 2^{-n-1} \leq 2^{-l_{k}} \leq 2^{-k}
\end{aligned}
$$

Hence, $A$ is Kurtz $h$-dimensional measure zero. 
Remark 2. By Theorem 2, we can give a triviality-type characterization of lowness for uniform Kurtz randomness via the following reducibility, although we hesitate to call this Kurtz reducibility, as it has a rather different form from Schnorr reducibility and $K$-reducibility. Let $A \leq_{\text {Kur }} B$ denote that, for each computable order $h$, the fact that $B$ is Kurtz $h$-dimensional measure zero implies that $A$ is Kurtz $h$-dimensional measure zero. If $A \leq_{\mathrm{Kur}} B$ and $A$ is Kurtz random, then $B$ is Kurtz random. A set $A \in 2^{\omega}$ is low for uniform Kurtz randomness if and only if $A \leq_{\text {Kur }} \emptyset$.

\section{Acknowledgement}

The first author was supported by a Grant-in-Aid for JSPS fellows. The second author was supported by GCOE, Kyoto University and JSPS KAKENHI 23740072 .

\section{References}

1. Barmpalias, G., Miller, J.S., Nies, A.: Randomness notions and partial relativization. Israel Journal of Mathematics pp. 1-26 (2011)

2. Bienvenu, L., Downey, R., Greenberg, N., Nies, A., Turetsky, D.: Characterizing lowness for Demuth randomness, submitted

3. Diamondstone, D., Greenberg, N., Turetsky, D.: A van lambalgen theorem for demuth randomness, submitted.

4. Downey, R., Griffiths, E.: Schnorr randomness. Journal of Symbolic Logic 69(2), $533-554(2004)$

5. Downey, R., Hirschfeldt, D.R.: Algorithmic Randomness and Complexity. Springer, Berlin (2010)

6. Downey, R.G., Griffiths, E.J., Reid, S.: On Kurtz randomness. Theoretical Computer Science 321, 249-270 (2004)

7. Franklin, J.N.Y., Stephan, F.: Schnorr trivial sets and truth-table reducibility. Journal of Symbolic Logic 75(2), 501-521 (2010)

8. Greenberg, N., Miller, J.S.: Lowness for Kurtz randomness. The Journal of Symbolic Logic 74, 665-678 (2009)

9. Hirschfeldt, D., Nies, A., Stephan, F.: Using random sets as oracles. Journal of the London Mathematical Society 75, 610-622 (2007)

10. Kurtz, S.A.: Randomness and Genericity in the Degrees of Unsolvability. Ph.D. thesis, University of Illinois at Urbana-Champaign (1981)

11. Miyabe, K.: Schnorr trivial and its equivalent notions, in preparation

12. Miyabe, K.: Truth-table Schnorr randomness and truth-table reducible randomness. Mathematical Logic Quarterly 57(3), 323-338 (2011)

13. Miyabe, K., Rute, J.: Van Lambalgen's Theorem for uniformly relative Schnorr and computable randomness, submitted.

14. Nies, A.: Lowness properties and randomness. Advances in Mathematics 197, 274$305(2005)$

15. Nies, A.: Computability and Randomness. Oxford University Press, USA (2009)

16. Stephan, F., Yu, L.: Lowness for weakly 1-generic and Kurtz-random. Lecture Notes in Computer Science 3959, 756-764 (2006)

17. Wang, Y.: Randomness and Complexity. Ph.D. thesis, University of Heidelberg (1996) 


\section{Appendix}

Proof (of (i) $\Longleftrightarrow$ (ii) of Proposition 1). (i) $\Rightarrow$ (ii): Let $f$ be a uniform Kurtz test. Then there exists $\psi: 2^{\omega} \times \omega \rightarrow\left(2^{<\omega}\right)<\omega$ such that $f(Z)=\bigcup_{n} \llbracket \psi(Z, n) \rrbracket$. Since $\mu(f(Z))=1$ for each $Z \in 2^{\omega}$, we can effectively calculate $t(Z, n)$ such that $\mu\left(\bigcup_{m \leq t(Z, n)} \llbracket \psi(Z, m) \rrbracket\right) \geq 1-2^{-n}$ and $t(Z, n)<t(Z, n+1)$. Let $g: 2^{\omega} \times \omega \rightarrow$ $\left(2^{<\omega}\right)^{<\omega}$ be a computable function such that

$$
\llbracket g(Z, n) \rrbracket=2^{\omega} \backslash \bigcup_{m \leq t(Z, n)} \llbracket \psi(Z, m) \rrbracket .
$$

Then $g$ is a uniform Kurtz null test and

$$
N=2^{\omega} \backslash f(A)=2^{\omega} \backslash \bigcup_{n} \llbracket \psi(Z, n) \rrbracket=\bigcap_{n} \llbracket g(Z, n) \rrbracket .
$$

(ii) $\Rightarrow$ (i): Let $g$ be a uniform Kurtz null test. Let $\phi: 2^{\omega} \times \omega \rightarrow\left(2^{<\omega}\right)^{<\omega}$ be a computable function such that $\llbracket \phi(Z, n) \rrbracket=2^{\omega} \backslash \llbracket g(Z, n) \rrbracket$, and let $f: 2^{\omega} \rightarrow \tau$ be such that $f(Z)=\bigcup_{n} \llbracket \phi(Z, n) \rrbracket$. Then $f$ is a total computable function and $\mu(f(Z))=1$ for each $Z \in 2^{\omega}$, because

$$
2^{\omega} \backslash f(Z)=2^{\omega} \backslash \bigcup_{n} \llbracket \phi(Z, n) \rrbracket=\bigcap_{n} \llbracket g(Z, n) \rrbracket
$$

is null.

Proof ( of (i) $\Longleftrightarrow$ (ii) of Proposition 2). (i) $\Rightarrow$ (ii): Suppose that there is a uniform Kurtz null test $f$ such that $A \in \bigcap_{n} \llbracket f(B, n) \rrbracket$. We can assume that, for each $n$, all the strings in $f(Z, n)$ for $Z \in 2^{\omega}$ have the same length $g(n)$, where $g$ is a computable order. Let $m: 2^{\omega} \rightarrow \mathbb{R}$ be a function such that

$$
m(Z)=\sum_{n} \sum_{\sigma \in f(Z, 2 n+2)} 2^{-|\sigma|-(n+1)}=\sum_{n} 2^{n+1} \mu(\llbracket f(Z, 2 n+2) \rrbracket) .
$$

Since $\mu(\llbracket f(Z, n) \rrbracket) \leq 2^{-(2 n+2)}, m(Z) \leq 1$ and $m$ is computable. By the $\mathrm{KC}$ Theorem, there is an oracle prefix-free machine $M$ such that $\mu\left(\operatorname{dom} M^{Z}\right)=m(Z)$ for each $Z \in 2^{\omega}$ and $K_{M^{Z}}(\sigma) \leq|\sigma|-(n+1)$ for each $\sigma \in f(Z, 2 n+2)$. Let $h(n)=g(2 n+2)$. Since $A\lceil g(2 n+2) \in f(B, 2 n+2)$, we have

$$
K_{M^{B}}(A\lceil h(n)) \leq h(n)-(n+1)
$$

for each $n$.

(ii) $\Rightarrow$ (i): Suppose that the pair $A, B$ satisfies (ii) via an oracle prefix-free machine $M$ and a computable function $h$ Let

$$
f(Z, n)=\left\{\sigma \in 2^{h(n)}: K_{M^{Z}}(\sigma)<f(n)-n .\right\} .
$$

Then $\mu(\llbracket f(Z, n) \rrbracket) \leq 2^{-n}$. Since $Z \mapsto \mu\left(\operatorname{dom} M^{Z}\right)$ is computable, there is a total computable function $\phi: 2^{\omega} \rightarrow 2^{\omega}$ such that $\phi(Z)(n)=\operatorname{dom}\left(M^{Z}\right)(n)$ for each $Z \in 2^{\omega}$ and $n \in \omega$. Then $f(Z, n)$ is finite and $f$ is a computable function. Thus, $f$ is a uniform Kurtz null test. By the definition of $M$ and $f, A \in \bigcap_{n} \llbracket f(B, n) \rrbracket$. 
We now restate Remark 2 formally.

Definition 4. Let $A, B \in 2^{\omega}$. We say that $A$ is Kurtz reducible to $B$ (denoted by $A \leq_{\text {Kur }} B$ ) if, for each computable order $h$, the fact that $B$ is Kurtz $h$ dimensional measure zero implies that $A$ is Kurtz h-dimensional measure zero.

Proposition 3. If $A \leq_{\mathrm{Kur}} B$ and $A$ is Kurtz random, then $B$ is Kurtz random.

Proof. Suppose $B$ is not Kurtz random. Then $B$ is Kurtz id-dimensional measure zero where id is the identity function. By the assumption $A \leq_{\mathrm{Kur}} B, A$ is Kurtz $h$-dimensional measure zero. Thus, $A$ is not Kurtz random.

Proposition 4. A set $A \in 2^{\omega}$ is low for uniform Kurtz randomness if and only if $A \leq_{\text {Kur }} \emptyset$.

Proof. Suppose that $A$ is low for uniform Kurtz randomness. Then $A$ is Kurtz $h$-dimensional measure zero for all computable orders $h$. Thus, $A \leq_{\text {Kur }} \emptyset$.

Suppose that $A \leq_{\text {Kur }} \emptyset$. Let $h$ be a computable order. Since $\emptyset$ is Kurtz $h$ dimensional measure zero, $A$ is Kurtz $h$-dimensional measure zero. Since $h$ is arbitrary, $A$ is low for uniform Kurtz randomness. 